\title{
Performance Analysis of Relaying Systems With Fixed and Energy Harvesting Batteries
}

\author{
Arooj Mubashara Siddiqui, Leila Musavian ${ }^{\circledR}$, Member, IEEE, Sonia Aïssa ${ }^{\circledR}$, Senior Member, IEEE, \\ and Qiang $\mathrm{Ni}^{\circledR}$, Senior Member, IEEE
}

\begin{abstract}
This paper focuses on the performance evaluation of an energy harvesting (EH) equipped dual-hop relaying system for which the end-to-end signal-to-noise ratio (SNR) and the overall system throughput are analyzed. The transmitter and relay nodes are equipped with both fixed and EH batteries. The source for harvesting at the transmitter is the solar energy, and at the relay node, the interference energy in the radio frequency is the harvesting source. Time switching scheme is used at the relay to switch between $\mathrm{EH}$ and decoding information. Harvest-use approach is implemented, and we investigate the effects of the harvesting energy in enhancing the performance of the relaying system by deriving estimated closed-form expressions for the cumulative distribution function of each link's individual SNR and of the end-to-end SNR. The analytical expression for the ergodic capacity is also derived. These expressions are validated through Monte-Carlo simulations. It is also shown that with the additional EH at the transmitter (source and relay), a significant improvement in the system throughput can be achieved when fixed batteries are running on low powers.
\end{abstract}

Index Terms- $\mathrm{EH}$, radio frequency $\mathrm{EH}$, solar $\mathrm{EH}$, relaying, time switching, cumulative distribution function.

\section{INTRODUCTION}

$\mathbf{T}$ HE continuously growing demand for higher data rates and the increase in the number of mobile devices have led to a rapid growth in the data traffic and communications infrastructure. This excessive demand in communicating data requires increasing energy consumption, which in turn, results in higher greenhouse gas emission, higher pollution and higher energy costs [1]. On the other hand, while the wireless traffic is increasing rapidly, battery capacity is still limited. In fact, the battery advancement has been much slower than the need

Manuscript received February 21, 2017; revised June 19, 2017 and August 12, 2017; accepted August 15, 2017. Date of publication September 19, 2017; date of current version April 16, 2018. This work was supported by the UK EPSRC under Grant EP/N032268/1. The associate editor coordinating the review of this paper and approving it for publication was Y. Li. (Corresponding author: Leila Musavian.)

A. M. Siddiqui is with the Department of Electrical Engineering, COMSATS Institute of Information Technology, Islamabad 44000, Pakistan (e-mail: aroojmubashara@comsats.edu.pk).

L. Musavian is with the School of Computer Science and Electronic Engineering, University of Essex, Colchester CO4 3SQ, U.K. (e-mail: leila.musavian@essex.ac.uk).

S. Aïssa is with the Institute National de la Recherche Scientifique (INRS-EMT), University of Quebec, Montreal, QC H5A 1K6, Canada (e-mail: aissa@emt.inrs.ca).

Q. $\mathrm{Ni}$ is with the InfoLab 21, School of Computing and Communications, Lancaster University, Lancaster LA1 4WA, U.K. (e-mail: q.ni@lancaster.ac.uk).

Color versions of one or more of the figures in this paper are available online at http://ieeexplore.ieee.org.

Digital Object Identifier 10.1109/TCOMM.2017.2754278 for long-life batteries. Hence, the gap between increasing the rate demands and the battery improvement had been widened over the last few years [1].

In addition to the battery limitation, according to the climate group SMART (2020) [2], higher greenhouse gas emissions have increased the carbon footprint to 349Mt and the electrical energy consumption to 1700TWh, which could result in loosing at least $5 \%$ of the global gross domestic product (GDP) every year. Due to the increase in the carbondioxide emission, limited battery life advancements, and the operational costs, several projects started to look for solutions to reduce the high energy consumption of communication networks [3], e.g., energy aware radio and network technologies (EARTH) [4] and towards real energy-efficient network design (TREND) [5]. There is an increasing interest in the academics and related industries to design and develop higher data rate devices while lowering the device energy consumption. As discussed in [6], and references therein, there are four key trade-offs between energy efficiency, spectrum efficiency, latency and deployment costs. In this regard, green wireless communication has a potential to provide possible solutions to the current energy limitation problems in the information and communication technology (ICT) sector [7].

\section{A. Motivation and Related Works}

Referring to the famous Shannon capacity formula, when the transmission power increases, the received signal-tonoise ratio (SNR) improves, and in turn, the link capacity increases [8]. The capacity may also increase when the distance between the communicating nodes decreases, which will effectively reduce the path loss [9]. Employing a relaying node between the transmitter and its end receiver, which reduces the distance between the communicating nodes, could improve the capacity. On the other hand, to increase the transmission power without spending more from the device fixed battery, one can employ an additional energy harvesting (EH) battery [10]. By implementing $\mathrm{EH}$ at the source transmitter and at the relay, the transmission rate can be improved while limiting the energy consumption from the fixed batteries [11].

$\mathrm{EH}$ has indeed the potential to prolong the lifetime and improve the performance of energy limited networks, e.g., wireless sensor networks (WSNs) [12], [13]. This technology not only promises to resolve the limited battery issues, but also to reduce the carbon footprint of high data rate wireless devices, by reusing the energy from the surrounding environ- 
ment [13]. Energy can be harvested from natural sources such as solar, thermal, wind and kinetic energy [1]. One of the most commonly used ambient energy sources is the solar energy, since light can be directly converted into electricity that runs a wide range of portable devices [14]. The solar energy is by far the largest and the most widely available source among the renewable energy sources. In a cellular network for example, the transmitter at the base station can have high solar energy panels [15].

Apart from conventional renewable sources, energy can also be harvested from ambient energy in the radio frequency (RF) [16]. The RF interference was used as a source of energy in many recent works [17]-[19]. In particular, the use of RF harvesting got popular due to the fact that it is autonomous and does not depend on dedicated energy sources [14], [16]. Among modern technologies manufacturers, we note Ambient Micro and Maxim who have developed methods for combining multiple EH resources [20]. Also, Powercast powerharvester devices convert the radio waves into DC power in order to increase the life-cycle of devices with low-power consumption [21].

On the other hand, due to practical circuit limitations, it is not possible to harvest energy and decode information at the same time [15], [22]. From an architectural point of view, there are two protocols for EH and wireless information transmission, e.g., time switching (TS) and power splitting (PS) [23]. In the former, the device switches over time to either harvest energy or to transmit information data. In PS, on the other hand, the receiver splits the received signal power into two parts, one for processing the information and the other for $\mathrm{EH}$ [23].

A solar EH point-to-point communication system was considered in [24] wherein the rate of the system was evaluated. A water-filling algorithm was proposed for improving the throughput of the communication links with harvest-store-use EH limited battery devices in [10]. Directional water-filling algorithms to maximize throughput in EH networks were later proposed in [25]. Optimum transmission policies under the constraints on energy causality condition of a fixed EH battery to maximise the throughput was studied in [26]. A comprehensive receiver architecture and rate-energy tradeoff analysis of an EH relaying system was presented in [27].

The combination of relaying and EH technology was shown useful in increasing the battery ife of relays in [23], in deploying WSNs in remote areas [28], and in multipleinput multiple-output (MIMO) networks [29]. Also, optimal network beamforming in collaborative relaying systems that uses both RF and solar EH was proposed in [35]. Optimal scheduling and power allocation for a two-hop relaying system with harvesting at the source and non-EH relays was developed in [36].

The received signal at the relay node is a combination of unwanted signals (interference) and the signal from the source [30]. We note that improving the signal-to-interferenceplus-noise ratio (SINR) by decreasing the interference is an important and challenging concern for most of the current communication networks [18]. In this field, various methods including multi-cell coordination or interference-alignment were used for interference cancellation [18]. Although interference reduces the throughput of the system, if it is used as a source of energy it may improve the system performance [30]. In fact, interference emerges as an additional source of energy that can help the communication process by improving the system performance [17]-[19], [30].

The performance analysis of RF EH relaying network was studied in [22], which considered energy constraint on the transmission power and transceiver hardware impairment for multiple EH relays. RF-powered WSNs with simultaneous wireless information and power transfer (SWIPT) was considered in [31], wherein stochastic geometry was used to analyze the performance on the downlink. Relay selection with residual impairments and multiple antennas devices was considered in [32], wherein the best relay was chosen using the channel-state-information (CSI) at each hop and applying maximal ratio combining (MRC). Similar performance analysis approach was used for non-orthogonal multiple access (NOMA) under Nakagami- $m$ fading in [33]. Also, cooperative NOMA with SWIPT, where the users close to the source act as EH relays, were considered in [34]. User selection schemes were proposed to improve the system throughput and outage probability in [34]. Outage probability and throughput of an amplify-and-forward relaying system using EH in Nakagami- $m$ fading channel is derived in [35]. In addition to that, performance analysis of a dual-hop underwater communication system subject to $\kappa-\mu$ shadowed fading channel with RF-EH was analyzed in [36].

Despite the importance of energy constraint and its effects on the transceiver design issues in cooperative networks, most of the research works in the literature focused on harvesting sources at the transmitter or at the relay with no fixed batteries. It is inevitable that a limited fixed battery is implemented within the communication system nodes [29]. Given the nature of the most types of EH sources, which is random and not necessarily available all the times, a limited fixed battery can provide extra flexibility and continuity in service. However, a thorough study on how EH can improve the performance of relaying system with fixed, but limited power batteries, is yet to be done.

\section{B. Contributions}

In this paper, a dual-hop relaying system, in which the source transmitter and the relay are both equipped with fixed, as well as, EH batteries is considered. The harvesting at the transmitter node is done from solar energy and the harvesting at the relay node is from RF interference. The TS scheme is used at the relay to harvest energy and to decode information. For the considered relaying system, we analyse and discuss the impact of EH on the system performance. First, closed-form expressions for the cumulative distribution function (CDF) of the end-to-end SNR, and also for each link SNRs are obtained. To do so, we analyse the randomness in the transmission power due to the RF interference energy at the relay, and we evaluate the end-to-end SNR. Analytical expression for the ergodic capacity is also derived. Numerical results for the 
validation of the developed analysis are obtained using MonteCarlo simulations. The effect of different parameters, such as the EH power, the fixed batteries power levels, and the energy conservation coefficient, are discussed and analysed.

To summarize, the main contributions of this paper are enlisted below:

- The benefits of having interference $\mathrm{EH}$ at the relay node and solar EH at the source transmitter, along with limited power fixed batteries, on the performance of a dualhop relaying system, are investigated. To the best of the authors' knowledge, this paper is the first to address this.

- Closed-form expressions for the CDF of the end-to-end SNR and the SNR at each link are derived. The closedform expressions reduce the computational complexity, but are also challenging to derive due to the presence of randomness in the interference at the relay node. Since, the channel between the relay and the sink is also random, the presence of multiplication of several random parameters in the received SNR, makes the analysis difficult. $^{1}$

- The analytical expression for the ergodic capacity, which is an important performance metric for delay-insensitive services, of the relaying system is obtained.

- The impact of the additional energy sources to improve the overall system performance is evaluated by using mathematical closed-form expressions, which are validated through Monte-Carlo simulations. Differences between the numerical results corresponding to the analysis and the Monte-Carlo simulations are small, indicating the correctness of the closed-form expressions.

- Finally, the impact of solar and interference EH level and also the fixed batteries power levels, on the system performance, is studied through numerical results.

\section{Organization}

The remainder of the paper is organized as follows. Section II describes the system model and assumptions. Performance analysis of the EH relaying system is discussed in Section III. In Section IV, we derive the CDF of the first-hop SINR, that of the second-hop SNR, and the one of the endto-end SNR. Results are expressed in closed-form. Also this section includes the derivation of the ergodic capacity. Finally, numerical results and discussion are given in Section $\mathrm{V}$, followed by the conclusion.

\section{System Model And Assumptions}

We consider a dual-hop decode-and-forward (DF) relaying system, in which the transmitter $T$ communicates with the sink $S$ through an EH relaying node $R$, as presented in Fig. 1. No direct communication between transmitter $T$ and the sink $S$ is possible, e.g., due to shadowing. Node $T$ is equipped with a fixed battery $B_{1}$ and a conventional solar EH battery. The relay node is also energy constrained with a fixed battery $B_{2}$

\footnotetext{
${ }^{1}$ The closed-form expressions developed here are of great help when it comes to the system design in practice, e.g., when the impact of interference, solar energy and energy conversion coefficient on the system performance needs to be investigated.
}

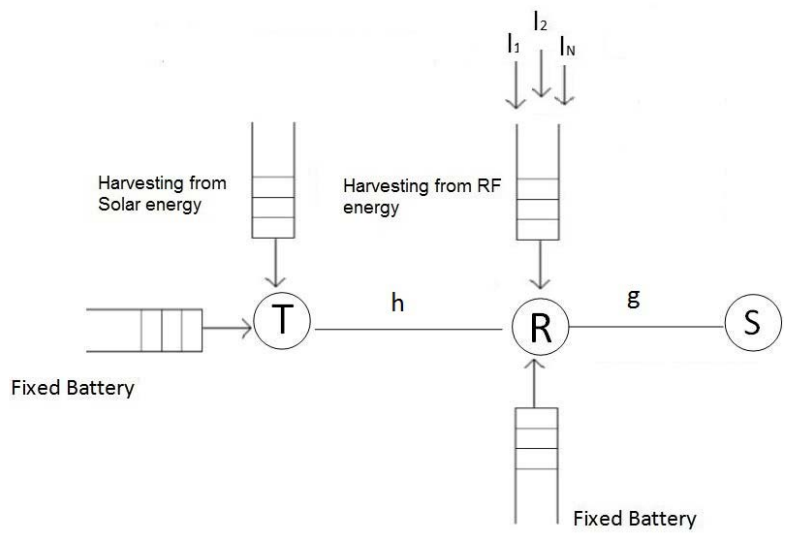

Fig. 1. Relaying system with fixed and energy-harvesting batteries.

but can also harvest energy from RF signals in the form of interference. The reason behind this assumption is that many current wireless networks, e.g., WSNs, operate on low and non-replaceable batteries [37]. The source can be a transmitter at high levels, and can thus benefit from solar EH, whereas the relay can be considered as a sensor node that operates on low and non-replaceable batteries. This system model is applicable to practical cases when we need to deploy WSNs for shortrange communications. This type of networks can indeed benefit from additional energy obtained through harvesting, even though this additional energy is not necessarily large. We note that, even if the fixed batteries are out of energy, the network may still be able to survive on EH batteries. The analysis and derivations of this paper can also apply to the system scenario where the fixed batteries are completely discharged, by simply replacing the value of the fixed battery level to zero in the paper's derivations.

The knowledge of the instantaneous channel state information (CSI) is not required at the source transmitter, as we are not considering adaptive power allocation at the source. When the relay node operates as a receiver during the first time slot, it is assumed to have the CSI of the link between the transmitter and the relay. However, instantaneous information of the interference channels are not known at the relay node. When the relay operates as a transmitter, it does not have knowledge of the instantaneous CSI of the link between the relay and the sink.

The power level of the transmitter and the relay fixed batteries are given by $P_{1}$ and $P_{2}$, respectively.

We also assume that the arrival rate of the harvesting solar energy at the source transmitter is constant, hence $Q_{\mathrm{t}}$ has a fixed value. Similar assumption is used in [38] where harvesting energy is assumed to have a continuous fashion, which is summed up to a constant value. Also, solar energy is considered in [39] which introduces a practical sensor node prototype that assumes solar energy to be constant for optimal event detection probability in the network. EH serving as the only source of energy, and modeled as a continuous function with fixed rate, is considered in [38] and a throughput maximization problem is solved in this paper.

The overall interference induced on the relay node originates from $N$ i.n.i.d. interferers [18], and is represented by $\sum_{i=1}^{N} I_{i}$, 


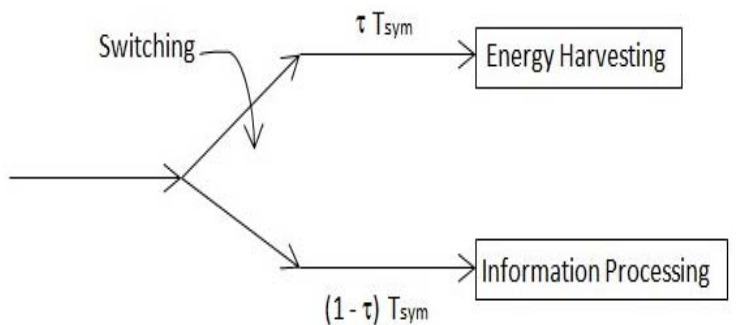

Fig. 2. Time-switching protocol for harvesting energy and information processing.

\section{Tsym}

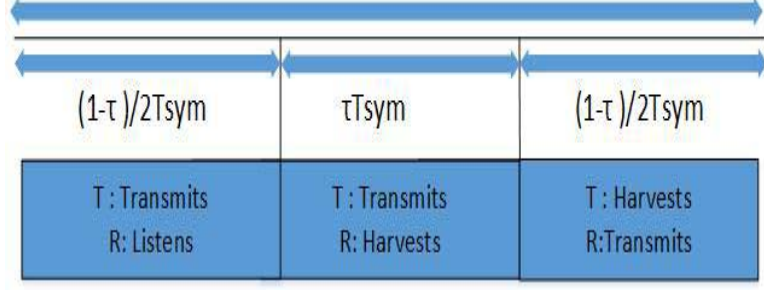

Fig. 3. Illustration of key parameters in time-switching protocol for harvesting energy and information processing.

where $I_{i}$ denotes the interference power of the $i$ th interferer. It is assumed that the interference and the desired channels are independent from each other. Here, the interference imposed on the other networks from the considered relaying system is not analysed. However, the results of this paper can pave the way for analysing a system with both incoming and imposing interference to the neighbouring networks. To do so, a further complex variable can be introduced for inter-cell interference. By that, the analysis and derivations of this paper can be further updated to be used for multi-cell applications.

We assume DF relaying is employed, hence, the message received at the relay node is decoded and then is forwarded to the sink. Each node is assumed to have a single antenna and to work in a half duplex mode within the dual-hop communication system. TS approach is used at the relay for EH and processing information, as described in Fig. 2. Let $T_{\text {sym }}$ denote the time during which the overall communication between the source transmitter and the sink takes place. $\tau T_{\text {sym }}$, for which $\tau$ varies from $0 \leq \tau \leq 1$, is the fraction of time in which the relay harvests energy both from the received signals and from the external interference. $(1-\tau) T_{\text {sym }}$ is then divided in two equal parts and represents the fraction of time during which information is transmitted from the source to the relay and from the relay to the sink. An illustration of the key parameters in the time allocation considered is provided in Fig. 3. In the TS architecture demonstrated in Fig. 3, the source node and the relay are assumed to have $\mathrm{HU}$ batteries, in which harvesting energy cannot be stored and must be used immediately when it becomes available for signal transmission. When the source is transmitting, the relay uses part of this time to listen and decode the source signal. When this phase finishes, the relay switches to harvesting. Since the relay cannot store the harvesting energy, the harvesting period is considered to be right before the relay signal transmission time. The relay harvests energy while the source is still transmitting. As soon as the harvesting energy becomes available at the relay, the latter will transmit the signal, which has already been decoded, to the sink destination. When relay is transmitting, the source node harvests energy, hence, no TS is required at the source transmitter.

In the considered HU approach, energy collected through harvesting is assumed available at the end of the harvesting time [39]. As there is no buffer to store the harvesting energy, the energy causality constraints are not applicable here. Rechargeable batteries, which consider energy causality constraints for energy storage through harvesting, are discussed in [40]. ${ }^{2}$ The EH circuit activation threshold, which defines the minimum energy level required to activate the circuit for harvesting [48], is considered $-10 \mathrm{dBm}$ for the relay node [37]. Additive white Gaussian noise (AWGN) is considered at the relay and at the sink.

\section{Channel Modeling Parameters}

The channel modeling parameters considered for the system model depend on different factors including path-loss, fading model and asymmetric characteristics. We start with path-loss description which mainly depends on the distance between the transmitter and the receiver, the operating frequency, and the environmental terrain [42]. We note that since the distance between the transmitter and the relay, i.e., $d_{R}$, is different from the distance between the relay and the sink, i.e., $d_{S}$, hence the path-loss factors of the first- and second-hop channels are different. Path-loss factor at first-hop is given by $P_{L_{R}}=$ $\mathrm{Kd}_{\mathrm{R}}^{-\beta}$, with $\mathrm{K}$ indicating a frequency dependent constant and $\beta>2$ referring to the environmental/terrain dependent pathloss exponent [42]. Similarly, $P_{L_{S}}$ represents the path-loss factor for the second-hop, which is given by $P_{L_{S}}=\mathrm{Kd}_{\mathrm{S}}^{-\beta}$. Note that the dual-hop relay considered in this paper is asymmetric and the path-loss factors for the channel between the transmitter and the relay is not necessarily the same as the one for the channel between the relay and the sink.

Channels follow the block flat-fading model, i.e., each of the channel gains are invariant during each fading block, but vary independently from one block to another. The length of each fading block is denoted by $T_{\mathrm{b}}$. The symbol duration is given by $T_{\text {sym }}=1 / B$, where $B$ is the system bandwidth. We assume that the fading block duration is an integer multiplication of the symbol duration. ${ }^{3}$ Furthermore, the fading block duration of all channels are assumed to be same.

The first-hop and second-hop channels experience independent Rayleigh fading and their respective channel power gains are given by $h$ and $g$ such that $h \sim C N\left(0, \Omega_{\mathrm{h}}\right)$ and $g \sim C N\left(0, \Omega_{\mathrm{g}}\right)$. Parameters $\Omega_{\mathrm{h}}$ and $\Omega_{\mathrm{g}}$ denote the variance of channel power gains $h$ and $g$, respectively.

\footnotetext{
${ }^{2}$ More information on the HU approach is provided in [41]. In the present work, the solar EH battery level is considered fixed within a transmission cycle, while the interference from the RF source is random given the nature of ambient energy. Similar to [39] and [41], no device equipment is dedicated to store energy.

${ }^{3}$ If $T_{\mathrm{b}}$ is smaller than $T_{\mathrm{sym}}$, then one symbol will be divided into two fading blocks and will experience different fading. Hence, the derivations of the paper will not hold anymore.
} 


\section{Performance Analysis of Energy-Harvesting RELAYING SYSTEM}

In this section, we evaluate the end-to-end SNR and the ergodic capacity of the relaying system with fixed and EH batteries, as described in Section II. The end-to-end ergodic capacity $C$ (in $\mathrm{b} / \mathrm{s} / \mathrm{Hz})$ is the average of the minimum between the rate at the first hop $\left(R_{1}\right)$ and the one at the second hop $\left(R_{2}\right)$, represented by

$$
C=\mathbb{E}\left(\min \left(R_{1}, R_{2}\right)\right),
$$

where $\mathbb{E}($.$) indicates the expectation operator and rates$ $R_{1}$ and $R_{2}$ are given by

$$
\begin{aligned}
& R_{1}=\frac{(1-\tau)}{2} \log _{2}\left(1+\gamma_{\mathrm{TR}}\right), \\
& R_{2}=\frac{(1-\tau)}{2} \log _{2}\left(1+\gamma_{\mathrm{RS}}\right),
\end{aligned}
$$

where $\gamma_{\mathrm{TR}}$ denotes the received SINR at the relay, and $\gamma_{\mathrm{RS}}$ indicates the received SNR at the sink. The effect of the interference at the relay is considered as an AWGN which is the worst effect of interference.

\section{A. First-Hop (Transmitter-to-Relay)}

During this phase, node $T$ transmits the information signal, consuming power $P_{1}$ from its fixed battery and an additional power $Q_{\mathrm{T}}$ from its EH battery. The SINR at the relay node can then be formulated as

$$
\gamma_{\mathrm{TR}}=\frac{\left(P_{1}+Q_{\mathrm{T}}\right) h}{K_{R}+P_{L_{R}} \sum_{i=1}^{N} I_{i}},
$$

where $K_{R}=P_{L_{R}} \sigma_{\mathrm{R}}^{2} B$, with $\sigma_{\mathrm{R}}^{2}$ indicating the variance of the AWGN and $Q_{\mathrm{T}}=\frac{(1-\tau) Q_{\mathrm{t}}}{1+\tau}$. The source transmitter-to-relay SINR, $\gamma_{\mathrm{TR}}$, is further simplified as

$$
\gamma_{\mathrm{TR}}=\frac{\gamma_{\mathrm{h}}}{1+I_{\mathrm{R}}}
$$

where $\gamma_{\mathrm{h}}=\frac{\left(P_{1}+Q_{\mathrm{T}}\right) h}{K_{R}}$ and $I_{\mathrm{R}}=\frac{\sum_{i=1}^{N} I_{i}}{\sigma_{\mathrm{R}}^{2} B}$. The received data is assumed to be decoded correctly only when the SINR is greater than a predefined threshold $\gamma$.

\section{B. Second-Hop (Relay-to-Sink)}

For the second-hop communication, the total power at the relaying node is the sum of the power from its fixed battery and the RF harvesting power from the interference and the source transmitter signal. Therefore, the power at the relay will be

$$
P_{\mathrm{R}}=P_{2}+Q_{\mathrm{R}}
$$

where $Q_{\mathrm{R}}$ indicates the power harvested at the relay node. ${ }^{4}$ The total energy that is harvested from the received information signal and from the interference signal for a duration

\footnotetext{
${ }^{4}$ As previously mentioned, $P_{2}$ is the power level of the relay's fixed battery $B_{2}$ and is assumed constant.
}

of $\tau T_{\text {sym }}$ at each block is given by

$$
E_{\mathrm{H}}=\eta\left[\sum_{i=1}^{N} I_{i}+\left(P_{1}+Q_{\mathrm{T}}\right) h\right] \tau T_{\mathrm{sym}},
$$

with $\eta$ indicating the energy conversion coefficient which varies from 0 to 1 [43]. The processing power at the relay, required by the transmit/receive circuitry is negligible compared to the power used for data transmission [30]. Therefore, we assume that the relay consumes the harvesting and the fixed battery energy for forwarding information to the sink. The transmission power at the relay can hence be written as

$$
Q_{\mathrm{R}}=\frac{E_{\mathrm{H}}}{(1-\tau) T_{\mathrm{sym}} / 2} .
$$

Replacing the value of $E_{\mathrm{H}}$ from (6) into (7) and substituting it into (5), yields

$$
P_{\mathrm{R}}=P_{2}+\frac{2 \tau \eta}{1-\tau}\left[\sum_{i=1}^{N} I_{i}+\frac{\left(P_{1}+Q_{\mathrm{T}}\right) h}{P_{L_{R}}}\right] .
$$

The SNR at the sink $S$ is hence given by

$$
\gamma_{\mathrm{RS}}=\frac{P_{\mathrm{R}} g}{K_{S}},
$$

where $K_{S}=P_{L_{S}} \sigma_{\mathrm{S}}^{2} B$, with $\sigma_{\mathrm{S}}^{2}$ indicating the variance of the AWGN at the sink. Inserting the value of $P_{\mathrm{R}}$ from (8) into $\gamma_{\mathrm{RS}}$ yields

$$
\gamma_{\mathrm{RS}}=\frac{\left[P_{2}+\frac{2 \tau \eta}{1-\tau}\left(\sum_{i=1}^{N} I_{i}+\frac{\left(P_{1}+Q_{\mathrm{T}}\right) h}{P_{L_{R}}}\right)\right] g}{K_{S}},
$$

which can be further simplified into

$$
\gamma_{\mathrm{RS}}=\gamma_{\mathrm{g}}+w\left(I_{\mathrm{R}}+\gamma_{\mathrm{h}}\right),
$$

where $\gamma_{\mathrm{g}}=\frac{g P_{2}}{K_{S}}$ and $w=\frac{2 \eta \tau \sigma_{\mathrm{R}}^{2} g}{\sigma_{\mathrm{S}}^{2} P_{L_{S}}(1-\tau)}$. We note that $w$ is a random variable (RV) with the same distribution as of $g$ but with a different variance.

\section{Closed-Form Derivations}

Here, we aim to derive a closed-form expression for the CDF of the transmitter-to-relay SINR, the relay-to-sink SNR, and also the end-to-end SNR for the considered EH DF relaying system with TS approach. By definition, the CDF of SNR at a certain threshold, $\gamma$, shows the probability of the instantaneous SNR to be less than $\gamma$. This threshold can represent the criterion for a minimum quality-of-service requirement at each node. The CDF of the SINR at the firsthop is formulated as $F_{\gamma_{\mathrm{TR}}}(\gamma)=\operatorname{Pr}\left(\gamma_{\mathrm{TR}} \leq \gamma\right)$, and the $\mathrm{CDF}$ of the SNR at the second-hop is given by $F_{\gamma_{\mathrm{RS}}}(\gamma)=\operatorname{Pr}\left(\gamma_{\mathrm{RS}} \leq \gamma\right)$, where $\operatorname{Pr}($.$) denotes the probability and F_{\mathrm{X}}(x)$ stands for the CDF of RV $X$ at $x$.

With the channels following independent Rayleigh fading, the probability distribution function (PDF) of $\gamma_{\mathrm{h}}$ is exponential and is given by

$$
f_{\gamma_{\mathrm{h}}}(x)=\frac{1}{\overline{\gamma_{\mathrm{h}}}} \exp \left(-\frac{x}{\overline{\gamma_{\mathrm{h}}}}\right), x \geq 0,
$$


where $\overline{\gamma_{\mathrm{h}}}$ is the average SNR from the source transmitter to the relay and is given by $\overline{\gamma_{\mathrm{h}}}=\frac{\left(P_{1}+Q_{\mathrm{T}}\right) \Omega_{\mathrm{h}}}{K_{R}}$.

Similarly, the PDF of the interference signal, $I_{\mathrm{R}}$, which is the sum of $N$ statistically i.n.i.d. exponential random interferences, each with a mean of $\mu_{i}$, can be written as [44]

$$
f_{I_{\mathrm{R}}}(y)=\sum_{i=1}^{v(\boldsymbol{A})} \sum_{j=1}^{\tau_{i}(\boldsymbol{A})} \lambda_{i j}(\boldsymbol{A}) \frac{\mu_{i}^{-j}}{(j-1) !} y^{j-1} \exp \left(\frac{-y}{\mu_{i}}\right), \quad y>0,
$$

where matrix $\boldsymbol{A}=\operatorname{diag}\left(\mu_{1}, \mu_{2}, \ldots, \mu_{N}\right)$, with $\mu_{1}>$ $\mu_{2}>\cdots>\mu_{v(A)}$ being the diagonal elements in decreasing order, $v(\boldsymbol{A})$ denotes the number of distinct diagonal elements of $\boldsymbol{A}, \tau_{i}(\boldsymbol{A})$ is the multiplicity of $\mu_{i}$, and $\lambda_{i j}$ is the $(i, j)$ th characteristic coefficient of $\boldsymbol{A}$ as discussed in [44].

\section{A. CDF of the First-Hop SINR}

The CDF of the first-hop received $\operatorname{SINR}, \operatorname{Pr}\left(\gamma_{\mathrm{TR}} \leq \gamma\right)$, can be expanded by inserting the value of $\gamma_{\mathrm{TR}}$ from (4) to get

$$
\operatorname{Pr}\left(\gamma_{\mathrm{TR}} \leq \gamma\right)=\operatorname{Pr}\left(\gamma_{\mathrm{h}} \leq\left(1+I_{\mathrm{R}}\right) \gamma\right)
$$

Using the PDF of $\gamma_{\mathrm{h}}$, given in (10), the CDF of the first-hop SINR for each value of interference $I_{\mathrm{R}}$ can be expanded as

$$
F_{\gamma_{\mathrm{TR}}}(\gamma)=\mathbb{E}_{I_{\mathrm{R}}}\left(1-\exp \left(-\gamma\left(\frac{1+I_{\mathrm{R}}}{\overline{\gamma_{\mathrm{h}}}}\right)\right)\right)
$$

where $\mathbb{E}_{I_{\mathrm{R}}}$ is the expectation operator with respect to $\mathrm{RV} I_{\mathrm{R}}$. The expression in (13) can be expanded by using the definition of expectation [45, p. 30], yielding

$$
F_{\gamma_{\mathrm{TR}}}(\gamma)=1-\int_{0}^{\infty} \exp \left(\frac{-\gamma(1+u)}{\overline{\gamma_{\mathrm{h}}}}\right) f_{I_{\mathrm{R}}}(u) d u .
$$

We now replace the PDF of interference $I_{\mathrm{R}}$ from (11) into (14) to get

$$
\begin{array}{r}
F_{\gamma_{\mathrm{TR}}}(\gamma)=1-\int_{0}^{\infty} \exp \left(\frac{-\gamma(1+u)}{\overline{\gamma_{\mathrm{h}}}}\right) \sum_{i=1}^{v(\boldsymbol{A})} \sum_{j=1}^{\tau_{i}(\boldsymbol{A})} \lambda_{i j}(\boldsymbol{A}) \\
\times \frac{\mu_{i}^{-j}}{(j-1) !} u^{j-1} \exp \left(\frac{-u}{\mu_{i}}\right) d u .
\end{array}
$$

In order to solve (15), we re-arrange the equation as

$$
\begin{aligned}
& F_{\gamma_{\mathrm{TR}}}(\gamma) \\
& =1-\sum_{i=1}^{v(\boldsymbol{A})} \sum_{j=1}^{\tau_{i}(\boldsymbol{A})} \exp \left(\frac{-\gamma}{\overline{\gamma_{\mathrm{h}}}}\right) \lambda_{i j}(\boldsymbol{A}) \\
& \quad \times \frac{\mu_{i}^{-j}}{(j-1) !}\left(\frac{1}{\mu_{i}}\left(1+\frac{\gamma \mu_{i}}{\overline{\gamma_{\mathrm{h}}}}\right)^{-1}\right) \\
& \quad \times \int_{0}^{\infty} \frac{u^{j-1}}{\mu_{i}}\left(1+\frac{\gamma \mu_{i}}{\overline{\gamma_{\mathrm{h}}}}\right) \exp \left(\frac{-u}{\mu_{i}}\left(1+\frac{\gamma \mu_{i}}{\overline{\gamma_{\mathrm{h}}}}\right)\right) d u .
\end{aligned}
$$

which can be solved in closed-form according to

$$
\begin{aligned}
& F_{\gamma_{\mathrm{TR}}}(\gamma) \\
& =1-\sum_{i=1}^{v(\boldsymbol{A})} \sum_{j=1}^{\tau_{i}(\boldsymbol{A})} \exp \left(\frac{-\gamma}{\overline{\gamma_{\mathrm{h}}}}\right) \lambda_{i j}(\boldsymbol{A}) \frac{\mu_{i}^{-j+1}}{(j-1) !} \\
& \quad \times\left(\left(1+\frac{\gamma \mu_{i}}{\overline{\gamma_{\mathrm{h}}}}\right)^{-1}(j-1) ! \mu_{i}^{j-1}\left(1+\frac{\gamma \mu_{i}}{\overline{\gamma_{\mathrm{h}}}}\right)^{-j+1}\right) .
\end{aligned}
$$

Therefore, the CDF of the first-hop received SINR is obtained by simplifying (17) to get

$$
F_{\gamma_{\mathrm{TR}}}(\gamma)=1-\sum_{i=1}^{v(\boldsymbol{A})} \sum_{j=1}^{\tau_{i}(\boldsymbol{A})} \exp \left(\frac{-\gamma}{\gamma_{\mathrm{h}}}\right) \lambda_{i j}(\boldsymbol{A})\left(1+\frac{\gamma \mu_{i}}{\gamma_{\mathrm{h}}}\right)^{-j} .
$$

The closed-form expressions for the CDF of the first-hop SINR, which are given in (18), depends on the average SNR from the transmitter to the relay $\left(\overline{\gamma_{\mathrm{h}}}\right)$ and on the mean value of each interference signal at the relay, e.g., $\mu_{i}, i=1, \cdots N$. (18) shows that the CDF of the first-hop SINR is directly related to $\mu_{i}, i=1, \cdots N$, whereas it is inversely related to $\overline{\gamma_{\mathrm{h}}}$. Hence, the CDF of the first-hop SINR decreases with the average SNR from the transmitter to the relay and increases with $\mu_{i}, i=1, \cdots N$.

\section{B. CDF of the Second-Hop SNR}

In this subsection, we obtain a closed-form expression for the $\mathrm{CDF}$ of the second-hop received SNR, $\operatorname{Pr}\left(\gamma_{\mathrm{RS}} \leq \gamma\right)$. The analysis is difficult due to the presence of randomness in the RF interference signals and in the fading channels. The transmit power at the relay follows a random exponential distribution according to (5). Since the channel between the relay and the sink is also random, the SNR will have the impact of these two random parameters multiplied.

We start by defining $z=\gamma_{\mathrm{h}}+I_{\mathrm{R}}$ to simplify (9). Then, the target CDF becomes

$$
\begin{aligned}
F_{\gamma_{\mathrm{RS}}}(\gamma) & =\operatorname{Pr}\left(\gamma_{\mathrm{g}}+w z \leq \gamma\right) \\
& =\mathbb{E}_{z} \mathbb{E}_{\gamma_{\mathrm{g}}}\left(1-\exp \left(-\frac{\gamma-\gamma_{\mathrm{g}}}{\bar{w} z}\right)\right),
\end{aligned}
$$

where $\bar{w}=\frac{2 \tau \eta \Omega_{\mathrm{g}} \sigma_{\mathrm{R}}^{2}}{\sigma_{\mathrm{S}}^{2} P_{L_{S}}(1-\tau)}, \mathbb{E}_{z}$ and $\mathbb{E}_{\gamma_{\mathrm{g}}}$ are the expectation operators with respect to RVs $z$ and $\gamma_{\mathrm{g}}$, respectively. We note that the PDF for $\gamma_{\mathrm{g}}$ is an exponential function given by

$$
f_{\gamma_{\mathrm{g}}}\left(\gamma_{\mathrm{g}}\right)=\frac{1}{\overline{\gamma_{\mathrm{g}}}} \exp \left(-\frac{\gamma_{\mathrm{g}}}{\overline{\gamma_{\mathrm{g}}}}\right)
$$

where $\overline{\gamma_{\mathrm{g}}}=\frac{P_{2} \Omega_{\mathrm{g}}}{K_{S}}$. In order to solve (19), we first solve the expectation with respect to $\gamma_{\mathrm{g}}$, to get

$$
\begin{aligned}
\operatorname{Pr}\left(\gamma_{\mathrm{g}}+w z \leq \gamma\right)=1- & \frac{1}{\overline{\gamma_{\mathrm{g}}}} \exp \left(\frac{-\gamma}{z \bar{w}}\right) \\
& \times \int_{0}^{\gamma} \exp \left(-\frac{\gamma_{\mathrm{g}}}{\overline{\gamma_{\mathrm{g}}}}+\frac{\gamma_{\mathrm{g}}}{\bar{w} z}\right) d \gamma_{\mathrm{g}} .
\end{aligned}
$$


Solving the integral in (21) and replacing it into (19) yields

$$
\begin{aligned}
& \operatorname{Pr}\left(\gamma_{\mathrm{g}}+w z \leq \gamma\right) \\
& =\mathbb{E}_{z}\left(\int _ { 0 } ^ { \infty } \left(1-\frac{\bar{w} z}{\gamma_{\mathrm{g}}-\bar{w} z} \exp \left(-\frac{\gamma}{\overline{\gamma_{\mathrm{g}}}}\right)\right.\right. \\
& \left.\left.\quad+\frac{\bar{w} z}{\gamma_{\mathrm{g}}-\bar{w} z} \exp \left(\frac{-\gamma}{z \bar{w}}\right)\right) f(z) d z\right) .
\end{aligned}
$$

Solving the expectation with respect to $z$ in (22) is challenging because the outer expectations $\mathbb{E}_{z}$ and inner expectation $\mathbb{E}_{\gamma_{\mathrm{g}}}$ are multiplied and the result becomes computationally difficult to solve. Recall that $z$ is defined as $z=\gamma_{\mathrm{h}}+I_{\mathrm{R}}$, which itself is a summation of two random variables with different distributions. Since $\gamma_{\mathrm{h}}$ and $I_{\mathrm{R}}$ are independent, we get the joint distribution, $f_{\gamma_{\mathrm{h}}, I_{\mathrm{R}}}(x, y)=f_{\gamma_{\mathrm{h}}}(x) f_{I_{\mathrm{R}}}(y)$. The PDF of $z$ is then calculated by inserting the individual PDFs, $f_{\gamma_{\mathrm{h}}}(x)$ and $f_{I_{\mathrm{R}}}(y)$, from (10) and (11), and integrating according to [30, p. 6428, eq. 13], which yields

$$
\begin{aligned}
f_{z}(z)= & \frac{1}{\overline{\gamma_{\mathrm{h}}}} \exp \left(\frac{-z}{\overline{\gamma_{\mathrm{h}}}}\right) \sum_{i=1}^{v(\boldsymbol{A})} \sum_{j=1}^{\tau_{i}(\boldsymbol{A})} \lambda_{i j}(\boldsymbol{A})\left(1-\frac{\mu_{i}}{\overline{\gamma_{\mathrm{h}}}}\right)^{-j} \\
& \times\left(1-\exp \left(-a_{i} z\right) \sum_{k=0}^{j-1} \frac{a_{i}^{k}}{k !} z^{k}\right),
\end{aligned}
$$

where $a_{i} \triangleq \frac{1}{\mu_{i}}-\frac{1}{\overline{\gamma_{\mathrm{h}}}}$. Then, by inserting the PDF of $z$ from (23) into (22), we obtain

$$
\begin{aligned}
& F_{\gamma_{\mathrm{RS}}}(\gamma) \\
& =1-\sum_{i=1}^{v(\boldsymbol{A})} \sum_{j=1}^{\tau_{i}(\boldsymbol{A})} \lambda_{i j}(\boldsymbol{A})\left(1-\frac{\mu_{i}}{\overline{\gamma_{\mathrm{h}}}}\right)^{-j} \\
& \times(\underbrace{\int_{\gamma}^{\infty} \frac{\bar{w} z}{\overline{\gamma_{\mathrm{h}}}\left(\gamma_{\mathrm{g}}-\bar{w} z\right)} \exp \left(\frac{-\gamma}{z \bar{w}}-\frac{z}{\overline{\gamma_{\mathrm{h}}}}\right) d z}_{J_{1}} \\
& +\underbrace{\frac{1}{\overline{\gamma_{\mathrm{h}}}} \exp \left(-\frac{\gamma}{\overline{\gamma_{\mathrm{g}}}}\right) \int_{\gamma}^{\infty} \frac{\bar{w} z}{\gamma_{\mathrm{g}}-\bar{w} z} \exp \left(\frac{-z}{\overline{\gamma_{\mathrm{h}}}}\right) d z}_{J_{2}} \\
& -\underbrace{\sum_{k=0}^{j-1} \frac{a_{i}^{k}}{k !} \int_{\gamma}^{\infty} \frac{1}{\overline{\gamma_{\mathrm{h}}}} \exp \left(-\frac{\gamma}{\bar{w} z}-\frac{z}{\overline{\gamma_{\mathrm{h}}}}\right) \exp \left(-a_{i} z\right) z^{k} d z}_{J_{3}}) .
\end{aligned}
$$

Since there is an additional fraction multiplied with the same entity in the exponential function present in the integral in (24), obtaining closed-form espression for (24) is challenging. We start by dividing (24) into three integrals, where the first and the second integrals are solved by using the generalized incomplete Gamma functions (given in [46]). Details are given below.

1) Closed-Form Expression for $J_{1}$ : The integral $J_{1}$ has a fractional equation of $z$ multiplied to an exponential function of $z$. We start with simplifying the ratio $\frac{\bar{w} z}{\gamma_{\mathrm{g}}-\bar{w} z}$ in (24), according to

$$
\begin{aligned}
\frac{\bar{w} z}{\gamma_{\mathrm{g}}-\bar{w} z} & =-\left(\frac{\gamma_{\mathrm{g}}-\gamma_{\mathrm{g}}-\bar{w} z}{\gamma_{\mathrm{g}}-\bar{w} z}\right) \\
& =-1+\left(1-\frac{\bar{w} z}{\gamma_{\mathrm{g}}}\right)^{-1},
\end{aligned}
$$

The term $\left(1-\frac{\bar{w} z}{\gamma_{\mathrm{g}}}\right)^{-1}$ can be approximated to $1+\frac{\bar{w} z}{\gamma_{\mathrm{g}}}$ for $\frac{\bar{w} z}{\gamma_{\mathrm{g}}} \rightarrow 0$. Thus, we have

$$
\frac{\bar{w} z}{\gamma_{\mathrm{g}}-\bar{w} z} \simeq \frac{\bar{w} z}{\gamma_{\mathrm{g}}}
$$

Now, we recall the formulation for generalized incomplete Gamma function given by [47, p. 372],

$$
\Gamma(\alpha, x ; b)=\int_{\alpha}^{\infty} t^{\alpha-1} \exp \left(-t-b t^{-1}\right) d t,
$$

which will later be used for obtaining a closed-form expression for $J_{1}$.

Lemma 1: Using the recurrence relation for the incomplete generalized Gamma function in [48, p. 101], the following equality can be obtained

$$
\begin{aligned}
\Gamma(\alpha+1, x ; b)=\alpha \Gamma & (\alpha, x ; b)+b \Gamma(\alpha-1, x ; b) \\
& +x^{\alpha} \exp \left(-x-b x^{-1}\right), \quad \alpha \geqslant 0 .
\end{aligned}
$$

Proof: The proof is provided in Appendix A.

Using the results of Lemma 1 for $\alpha=1$ and (26), a solution for the first integral $J_{1}$ can be obtained according to

$$
\begin{aligned}
& J_{1} \simeq \frac{\overline{\gamma_{\mathrm{g}}}}{\overline{\gamma_{\mathrm{g}} \gamma_{\mathrm{h}}}}\left(\Gamma\left(0, \frac{\gamma}{\overline{\gamma_{\mathrm{h}}}} ; \frac{\gamma}{\overline{\gamma_{\mathrm{h}} \gamma_{\mathrm{g}} w}}\right)\right. \\
& \left.\quad-\gamma \exp \left(-\gamma-b \gamma^{-1}\right)+\Gamma\left(1, \frac{\gamma}{\overline{\gamma_{\mathrm{h}}}} ; \frac{\gamma}{\overline{\gamma_{\mathrm{h}} \gamma_{\mathrm{g}} w}}\right)\right) .
\end{aligned}
$$

2) Closed-Form Expression for $J_{2}$ : To obtain a closed-from expression for $J_{2}$ (shown in (24)), we use the approximation result in (26), yielding

$$
J_{2} \simeq \frac{\bar{w}}{\overline{\gamma_{\mathrm{h}} \gamma_{\mathrm{g}}}} \exp \left(-\frac{\gamma}{\overline{\gamma_{\mathrm{g}}}}\right) \int_{\gamma}^{\infty} z \exp \left(\frac{-z}{\overline{\gamma_{\mathrm{h}}}}\right) d z
$$

which allows us to obtain a closed-form expression for $J_{2}$ as

$$
J_{2} \simeq-\frac{\bar{w}}{\overline{\gamma_{\mathrm{h}} \gamma_{\mathrm{g}}}}\left(1-\frac{\gamma}{\overline{\gamma_{\mathrm{h}}}}\right) \exp \left(-\gamma\left(\frac{1}{\overline{\gamma_{\mathrm{g}}}}+\frac{1}{\overline{\gamma_{\mathrm{h}}}}\right)\right) .
$$

3) Closed-Form Expression for $J_{3}$ : Using (27), we obtain a closed-form expression for $J_{3}$, according to,

$$
\begin{aligned}
J_{3}=\frac{1}{\overline{\gamma_{\mathrm{h}}}} \exp & \left(a_{i}\right) \sum_{k=0}^{j-1} \frac{1}{k !}\left(-a_{i}\right)^{k} \\
& \times \sum_{m=0}^{k}\left(\begin{array}{l}
k \\
m
\end{array}\right) \frac{b_{i}^{-1}}{\left(-b_{i}\right)^{m}} \Gamma\left(m+1, b_{i} \gamma ; \frac{b_{i} \gamma}{\bar{w}}\right),
\end{aligned}
$$

where $b_{i}=\frac{1}{\overline{\gamma_{\mathrm{h}}}}+\frac{a_{i}}{1+\gamma}$ and $a_{i}$ is defined right after (23). 
Finally, we obtain the closed-form solution for the CDF of $F_{\gamma_{\mathrm{RS}}}(\gamma)$, by inserting (29), (30) and (31) into (24), yielding

$$
\begin{aligned}
& F_{\gamma_{\mathrm{RS}}}(\gamma) \\
& \simeq 1-\sum_{i=1}^{v(\boldsymbol{A})} \sum_{j=1}^{\tau_{i}(\boldsymbol{A})} \lambda_{i j}(\boldsymbol{A})\left(1-\frac{\mu_{i}}{\overline{\gamma_{\mathrm{h}}}}\right)^{-j} \\
& \quad \times \frac{\bar{w}}{\overline{\gamma_{\mathrm{g}}}}\left(-\gamma \exp \left(-\gamma-b \gamma^{-1}\right)+\Gamma\left(1, \frac{\gamma}{\overline{\gamma_{\mathrm{h}}}} ; \frac{\gamma}{\overline{\gamma_{\mathrm{h}} \gamma_{\mathrm{g}} w}}\right)\right. \\
& \left.\quad+\Gamma\left(0, \frac{\gamma}{\overline{\gamma_{\mathrm{h}}}} ; \frac{\gamma}{\overline{\gamma_{\mathrm{h}} \gamma_{\mathrm{g}} w}}\right)\right)-\frac{\bar{w}}{\overline{\gamma_{\mathrm{g}} \gamma_{\mathrm{h}}}}\left(1-\frac{\gamma}{\overline{\gamma_{\mathrm{h}}}}\right) \\
& \quad \times \exp \left(-\gamma\left(\frac{1}{\overline{\gamma_{\mathrm{h}}}}+\frac{1}{\overline{\gamma_{\mathrm{g}}}}\right)\right)-\frac{1}{\overline{\gamma_{\mathrm{h}}}} \exp \left(a_{i}\right) \sum_{k=0}^{j-1} \frac{1}{k !}\left(-a_{i}\right)^{k} \\
& \quad \times \sum_{m=0}^{k}\left(\begin{array}{c}
k \\
m
\end{array}\right) \frac{b_{i}^{-1}}{\left(-b_{i}\right)^{m}} \Gamma\left(m+1, b_{i} \gamma ; \frac{b_{i} \gamma}{\bar{w}}\right) .
\end{aligned}
$$

\section{CDF of the End-to-End SNR}

The end-to-end SNR is defined by the probability that the instantaneous output SNR falls below a certain threshold which is already defined as $\gamma$. Mathematically, it is written as

$$
\operatorname{Pr}\left(\min \left(\gamma_{\mathrm{TR}}, \gamma_{\mathrm{RS}}\right) \leq \gamma\right)=\operatorname{Pr}\left(\left(\gamma_{\mathrm{g}}+w z\right) \mathbb{1} \leq \gamma\right)
$$

where $\mathbb{1}$ symbolizes an indicator of RV, with $\mathbb{1}=1$ for $\gamma_{\mathrm{TR}}>\gamma$ and 0 otherwise. This RV also indicates that the power at the relay is not only the power coming from its fixed battery $B_{2}$, but also the random replenished energy from the interference. Let us introduce the $\operatorname{RV} \hat{z}=\left(\gamma_{\mathrm{h}}+I_{\mathrm{R}}\right) \mathbb{1}$. The PDF $f_{z}(\hat{z})$ is given by

$$
\begin{aligned}
f_{z}(\hat{z})= & \mathbb{1} \frac{1}{\overline{\gamma_{\mathrm{h}}}} \exp \left(\frac{-\hat{z}}{\overline{\gamma_{\mathrm{h}}}}\right) \sum_{i=1}^{v(\boldsymbol{A})} \sum_{j=1}^{\tau_{i}(\boldsymbol{A})} \lambda_{i j}(\boldsymbol{A})\left(1-\frac{\mu_{i}}{\overline{\gamma_{\mathrm{h}}}}\right)^{-j} \\
& \times\left(1-\exp \left(-a_{i} \frac{\hat{z}-\gamma}{1+\gamma}\right) \sum_{k=0}^{j-1} \frac{a_{i}^{k}}{k !}\left(\frac{\hat{z}-\gamma}{1+\gamma}\right)^{k}\right) .
\end{aligned}
$$

Thus, by inserting (34) into (22), the PDF of the end-toend SNR, $\gamma_{\text {end-to-end, is obtained as Obtaining a closed-form }}$ solution for (35), as shown at the bottom of this page, can be done by using a similar method as in subsection IV-B. It is noted that the solutions to the first and the second integrals are already known from (29) and (30). In the third integral, we use the Taylor series expansion for $(\hat{z}-\gamma)^{k}$ [49] and use the definition of generalized incomplete Gamma function (given in [47, p. 372]). Details are given below.

Closed-Form Expression for $K_{3}$ : The third integral $K_{3}$ is solved by using direct substitution of a Taylor series expansion of $(\hat{z}-\gamma)^{k}$ with respect to $\hat{z}[30$, p. 6430] and the identity of the generalized incomplete Gamma function [46, eq. (3.351.1), eq.(3.334)]. This gives

$$
\begin{aligned}
K_{3} \simeq \frac{1}{\gamma_{\mathrm{h}}} & \exp \left(\frac{a_{i} \gamma}{1+\gamma}\right) \sum_{k=0}^{j-1} \frac{1}{k !}\left(\frac{-a_{i} \gamma}{1+\gamma}\right)^{k} \\
& \times \sum_{m=0}^{k}\left(\begin{array}{l}
k \\
m
\end{array}\right) \frac{b_{i}^{-1}}{\left(-b_{i} \gamma\right)^{m}} \Gamma\left(m+1, b_{i} \gamma ; \frac{b_{i} \gamma}{\overline{\gamma_{\mathrm{g}} w}}\right) .
\end{aligned}
$$

We now obtain the closed-form solution for the CDF of the end-to-end SNR by inserting (29), (30) and (36), into (24), yielding

$$
\begin{aligned}
F_{\gamma_{\text {end-to-end }}}(\gamma) \\
\simeq 1-\sum_{i=1}^{v(\boldsymbol{A})} \sum_{j=1}^{\tau_{i}(\boldsymbol{A})} \lambda_{i j}(\boldsymbol{A})\left(1-\frac{\mu_{i}}{\overline{\gamma_{\mathrm{h}}}}\right)^{-j} \\
\times \frac{\bar{w}}{\overline{\gamma_{\mathrm{g}}}}\left(-\gamma \exp \left(-\gamma-b \gamma^{-1}\right)+\Gamma\left(1, \frac{\gamma}{\overline{\gamma_{\mathrm{h}}}} ; \frac{\gamma}{\overline{\gamma_{\mathrm{h}} \gamma_{\mathrm{g}} w}}\right)\right. \\
\left.+\Gamma\left(0, \frac{\gamma}{\overline{\gamma_{\mathrm{h}}}} ; \frac{\gamma}{\overline{\gamma_{\mathrm{h}} \gamma_{\mathrm{g}} w}}\right)\right)-\frac{\bar{w}}{\overline{\gamma_{\mathrm{g}} \gamma_{\mathrm{h}}}}\left(1-\frac{\gamma}{\overline{\gamma_{\mathrm{h}}}}\right) \\
\times \exp \left(-\gamma\left(\frac{1}{\overline{\gamma_{\mathrm{h}}}}+\frac{1}{\overline{\gamma_{\mathrm{g}}}}\right)\right)-\frac{1}{\overline{\gamma_{\mathrm{h}}}} \exp \left(\frac{a_{i} \gamma}{1+\gamma}\right) \\
\quad \times \sum_{k=0}^{j-1} \frac{1}{k !}\left(\frac{-a_{i} \gamma}{1+\gamma}\right)^{k} \sum_{m=0}^{k}\left(\begin{array}{c}
k \\
m
\end{array}\right) \frac{b_{i}^{-1}}{\left(-b_{i} \gamma\right)^{m}} \\
\times \Gamma\left(m+1, b_{i} \gamma ; \frac{b_{i} \gamma}{\overline{\gamma_{\mathrm{g}} w}}\right) .
\end{aligned}
$$

We note that the transmission power at the relay, i.e., $P_{\mathrm{R}}$, does not only depend on the power level of the relay's fixed battery, but also on the energy available through harvesting. We recall

$$
\begin{aligned}
F_{\gamma_{\text {end-to-end }}(\gamma)=} & -\sum_{i=1}^{v(\boldsymbol{A})} \sum_{j=1}^{\tau_{i}(\boldsymbol{A})} \lambda_{i j}(\boldsymbol{A})\left(1-\frac{\mu_{i}}{\overline{\gamma_{\mathrm{h}}}}\right)^{-j}(\underbrace{\int_{\gamma}^{\infty} \frac{1}{\overline{\gamma_{\mathrm{h}}}} \frac{\bar{w} \hat{z}}{\gamma_{\mathrm{g}}-\bar{w} \hat{z}} \exp \left(\frac{-\gamma}{\hat{z} \bar{w}}-\frac{\hat{z}}{\overline{\gamma_{\mathrm{h}}}}\right) d \hat{z}}_{K_{1}} \\
& +\underbrace{\frac{1}{\overline{\gamma_{\mathrm{h}}}} \exp \left(-\frac{\gamma}{\overline{\gamma_{\mathrm{g}}}}\right) \int_{\gamma}^{\infty} \frac{\bar{w} \hat{z}}{\gamma_{\mathrm{g}}-\bar{w} \hat{z}} \exp \left(\frac{-\hat{z}}{\overline{\gamma_{\mathrm{h}}}}\right) d \hat{z}}_{K_{2}} \\
& -\underbrace{\sum_{k=0}^{j-1} \frac{a_{i}^{k}}{k !} \int_{\gamma}^{\infty} \frac{1}{\overline{\gamma_{\mathrm{h}}}} \exp \left(-\frac{\gamma}{\bar{w} \hat{z}}-\frac{\hat{z}}{\overline{\gamma_{\mathrm{h}}}}\right)-\exp \left(-a_{i} \frac{\hat{z}-\gamma}{1+\gamma}\right)\left(\frac{\hat{z}-\gamma}{1+\gamma}\right)^{k} d \hat{z}}_{K_{3}}) .
\end{aligned}
$$


TABLE I

Simulation PARAMETERS

\begin{tabular}{|c|c|c|c|}
\hline Parameters & Default value & Parameters & Default value \\
\hline$N$ & 5 & $\eta$ & 0.3 else stated \\
\hline$\gamma$ & $0.2 \mathrm{~dB}$ & $\mu_{i}, i=1, \ldots, 5$ & 0.3 \\
\hline$\tau$ & 0.4 else stated & $\sigma_{\mathrm{S}}^{2} B$ & 1 else stated \\
\hline$P$ & $1 \mathrm{~dB}$ else stated & $K_{\mathrm{R}}$ & 1 else stated \\
\hline$\Omega_{h}$ & 1 else stated & $\sigma_{\mathrm{R}}^{2} B$ & 1 else stated \\
\hline$K_{\mathrm{S}}$ & 1 else stated & $\Omega_{g}$ & 1 else stated \\
\hline
\end{tabular}

that $\mathrm{EH}$ at the relay node is the combination of the energy collected from interference and the energy received from the source's signals. Hence, the distribution of the received SNR at the sink node depends on the variations of the relay-to-sink, as well as the transmitter-to-relay, channel power gains. One can show that the second-hop SNR can be improved if the level of interference increases. However, the effect of the interference power on the end-to-end SNR, given in (37), cannot be easily verified. This can also be intuitively explained since in the considered relaying system, the interference reduces the SINR of the first hop, whereas it increases the SNR of the second hop.

\section{Ergodic Capacity}

In this subsection, we analytically derive the ergodic capacity of the relaying system which is calculated by taking the average of the minimum between the rate at the first-hop and that at the second-hop [50], formulated in $\mathrm{b} / \mathrm{s} / \mathrm{Hz}$ as

$$
\begin{aligned}
C= & \mathbb{E}\left(\min \left(\frac{(1-\tau)}{2} \log _{2}\left(1+\gamma_{\mathrm{TR}}\right), \frac{(1-\tau)}{2} \log _{2}\left(1+\gamma_{\mathrm{RS}}\right)\right)\right) \\
= & \mathbb{E}\left(\frac{1-\tau}{2} \log _{2}\left(1+\min \left(\gamma_{\mathrm{TR}}, \gamma_{\mathrm{RS}}\right)\right)\right) \\
= & \frac{1-\tau}{2} \int_{0}^{\infty} \log _{2}\left(1+\gamma_{\text {end-to-end }}\right) \\
& \times f_{\gamma_{\text {end-to-end }}}\left(\gamma_{\text {end-to-end }}\right) \mathrm{d} \gamma_{\text {end-to-end }},
\end{aligned}
$$

where $f_{\gamma_{\text {end-to-end }}(.)}$ is the $\mathrm{PDF}$ of the $\mathrm{RV} \gamma_{\text {end-to-end }}=$ $\min \left(\gamma_{\mathrm{TR}}, \gamma_{\mathrm{RS}}\right)$. We now solve (38) by using integration by parts as follows:

$$
\begin{aligned}
C= & \frac{1-\tau}{2}\left(\log _{2}\left(1+\gamma_{\text {end-to-end }}\right)\right. \\
& \left.\times\left(F_{\gamma_{\text {end-to-end }}}\left(\gamma_{\text {end-to-end }}\right)-1\right)\right)\left.\right|_{0} ^{\infty}-\frac{1-\tau}{2 \ln 2} \\
& \times \int_{0}^{\infty} \frac{F_{\gamma_{\text {end-to-end }}}\left(\gamma_{\text {end-to-end }}\right)-1}{1+\gamma_{\text {end-to-end }}} d \gamma_{\text {end-to-end }} \\
= & \frac{1-\tau}{2 \ln 2} \int_{0}^{\infty} \frac{1-F_{\gamma_{\text {end-to-end }}}\left(\gamma_{\text {end-to-end }}\right) d \gamma_{\text {end-to-end }}}{1+\gamma_{\text {end-to-end }}} .
\end{aligned}
$$

where, $F_{\gamma_{\text {end-to-end }}}\left(\gamma_{\text {end-to-end }}\right)$ is given in (37).

\section{Numerical Results AND Discussion}

In this section, we numerically evaluate the effect of $\mathrm{EH}$, the fixed batteries power levels and the energy conversion coefficient on the ergodic capacity and the end-to-end SNR of the studied relaying system. In all the figures, we assume that the power of the fixed battery at the source transmitter $T$



Fig. 4. The CDF of $\gamma_{\text {TR }}$ (first-hop), CDF of $\gamma_{\mathrm{RS}}$ (second-hop), and the CDF of $\gamma_{\text {end-to-end }}$ (end-to-end SNR) versus the power consumed from the fixed batteries $P$, when $\gamma=0.2, \eta=0.3, K_{R}=0.5$ and $K_{S}=0.3$ and $Q_{\mathrm{t}}=1 \mathrm{~dB}$.

is equal to the power of the fixed battery at the relay $R$, hence, $P_{1}=P_{2}=P$. In our simulation we have used values directly for $K_{R}$, e.g., $K_{R}=1$, in which the effect of distance is included through the path-loss model. Hence we did not choose any specific value of $d_{R}$. In the simulations, we included the value for $K_{R}=K_{S}=1$, else stated. The channels are assumed unit variance Rayleigh, hence, $\Omega_{\mathrm{h}}=\Omega_{\mathrm{g}}=1$, unless otherwise indicated. The number of interference signals at the relay is set to $N=5$ with normalized $\mu_{i}=0.3$, $i=1, \ldots 5$, and the TS parameter is set to $\tau=0.4$, unless otherwise indicated. Values for $Q_{\mathrm{t}}$ and $\eta$ are taken from studies shown in [51]. The noise variance at relay and sink is assumed to be equal, hence $\sigma_{\mathrm{R}}^{2}=\sigma_{\mathrm{S}}^{2}=1$, unless otherwise indicated. Table I demonstrates the values for the parameters considered for the simulations.

In Fig. 4, we start by plotting the CDF of the first-hop SINR $F_{\gamma_{\mathrm{TR}}}(\gamma)$, the CDF of the second-hop SNR $F_{\gamma_{\mathrm{RS}}}(\gamma)$, and the CDF of the end-to-end SNR $F_{\gamma_{\text {end-to-end }}}(\gamma)$ versus the power consumed from the fixed batteries $P$, at $\gamma=0.2$, $\eta=0.3, K_{R}=0.5$ and $K_{S}=0.3$ with $Q_{\mathrm{t}}=1 \mathrm{~dB}$. The figure includes both analytical and Monte-Carlo simulation results to verify the correctness of the closed-form derivations. From the graphs, we observe that the CDF of the received SNR at the second hop is greater than the one of the SINR at the first hop, for most of the time. It is noted that the CDF of the end-to-end SNR is similar to the CDF of the second hop SNR $F_{\gamma_{\mathrm{RS}}}(\gamma)$. This is due to the fact that, for the settings used in this figure, the first hop SINR is the minimum of the SINR at the first and the SNR at the second hop for most of the time. Further, the plots confirm that the results obtained from the closed-form expressions match to the ones obtained from the Monte-Carlo simulations, hence indicating that the obtained closed-form expressions yield accurate measures of the CDF of the individual links' SNRs and the end-to-end SNR.

Fig. 5 shows the plots for $\mathbb{E}\left(R_{1}\right)$ (the average rate at the first hop) and $\mathbb{E}\left(R_{2}\right)$ (the average rate at the second hop) versus the power level of the fixed batteries $P$, at $\eta=0.3$ and for various values of $Q_{\mathrm{t}}$. From the plots, we note that when $P=0 \mathrm{~dB}$ and no harvesting is available at the source, the average rate of the second hop is slightly higher than the one at the first hop. The relay harvests energy both from source signal 




Fig. 5. Average rates at the first and second hops $\left(\mathbb{E}\left(R_{1}\right), \mathbb{E}\left(R_{2}\right)\right)$ versus $P$ with $\eta=0.3$.

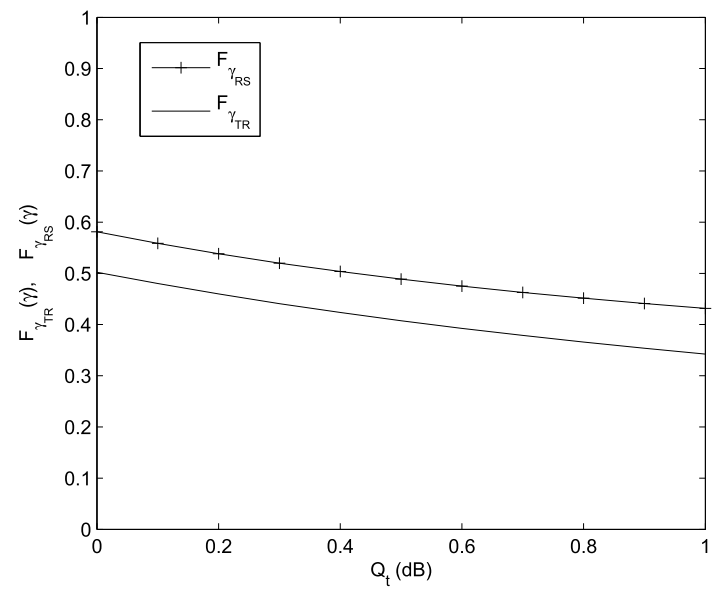

Fig. 6. $\quad F_{\gamma_{\mathrm{TR}}}(\gamma)$ and $F_{\gamma_{\mathrm{RS}}}(\gamma)$ versus $Q_{\mathrm{t}}$ at $\gamma=0.2$ with $\eta=0.3$ and $P=1 \mathrm{~dB}$.

and RF interference and, hence, under the above-mentioned conditions, the second hop achieves a slightly higher rate than the first hop. When $Q_{\mathrm{t}}=1 \mathrm{~dB}$, and at the same time the energy conversion coefficient at the relay remains small $(\eta=0.3)$, the average rate at the first hop $\mathbb{E}\left(R_{1}\right)$ dominates $\mathbb{E}\left(R_{2}\right)$. This happens because the solar energy at the transmitter is stronger compared to the RF energy level at the relay. We also observe that the difference between the two rates decreases at higher power values $P$ of the fixed batteries, indicating that $\mathrm{EH}$ is less beneficial in devices with higher powers.

Fig. 6 includes the plots for the CDF of the SINR at the first-hop $\gamma_{\mathrm{TR}}$ and the CDF of the SNR at the second hop $\gamma_{\mathrm{RS}}$, versus $Q_{\mathrm{t}}$, at $\eta=0.3, P=1 \mathrm{~dB}$ and $\gamma=0.2$. From the graph, we notice that not only the CDF of the first hop SINR $\left(F_{\gamma_{\text {TR }}}(\gamma)\right)$ decreases with the increase in the solar energy, but also the $\operatorname{CDF}$ of the second hop $\operatorname{SNR}\left(F_{\gamma_{\mathrm{RS}}}(\gamma)\right)$ shows a similar trend.

Fig. 7 illustrates the plots for the average rates $\mathbb{E}\left(R_{1}\right)$, $\mathbb{E}\left(R_{2}\right)$ and $C$ versus the TS parameter $\tau$ with $\eta=0.3$, $\mu=0.3, P=1 \mathrm{~dB}$, and various values of $Q_{\mathrm{t}}$. It is noted that when $Q_{\mathrm{t}}=0.5 \mathrm{~dB}, \mathbb{E}\left(R_{2}\right)$ shows bell curve while $\mathbb{E}\left(R_{1}\right)$ is monotonically decreasing. This is due to the fact that although with increasing $\tau$, more energy will be available at the relay node through harvesting, but at the same time, less time is available for signal information transmission. This conflicting

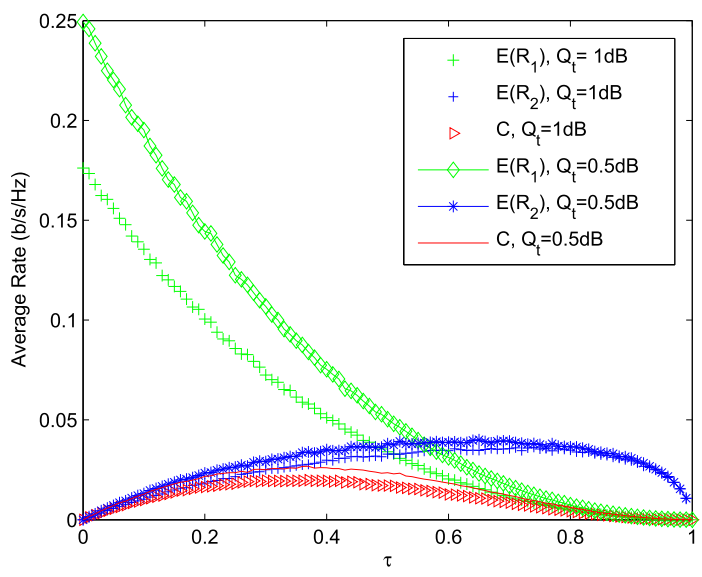

Fig. 7. Average rates at the first and second hops, $\left(\mathbb{E}\left(R_{1}\right), \mathbb{E}\left(R_{2}\right)\right)$, versus $\tau$, with $\eta=0.3$ and various values of $Q_{\mathrm{t}}$.

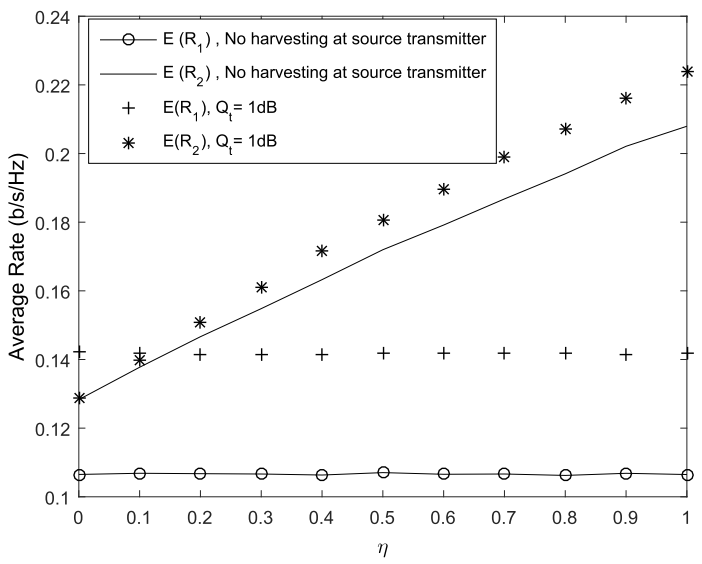

Fig. 8. Average rates (first-hop and second-hop) versus the energy conversion coefficient $\eta$, with $P=1 \mathrm{~dB}$ and various values of $Q_{\mathrm{t}}$.

effect, makes the shape of $\mathbb{E}\left(R_{2}\right)$ with respect to $\tau$ bell-shape. When solar energy increases, e.g., $Q_{\mathrm{t}}=1 \mathrm{~dB}$, the two curves coincide each other at smaller values of $\tau=0.5$. This happens because with higher $Q_{\mathrm{t}}, \mathbb{E}\left(R_{1}\right)$ is bigger. Hence, the amount of time which will be used for harvesting, instead of information transmission reduces $\mathbb{E}\left(R_{1}\right)$ quicker and hence, the two curve coincide at a smaller value of $\tau$, when compared to the case with $Q_{\mathrm{t}}=0.5 \mathrm{~dB}$. Generally, the trend shows the higher the solar energy is, the less time should be allocated for harvesting in the second hop.

In Fig. 8, we present the plots for the average rate at the first-hop and the average rate at the second-hop versus energy conversion coefficient $\eta$ at $P=1 \mathrm{~dB}$ with various values of $Q_{\mathrm{t}}$. At small values of $\eta$, i.e., $\eta<0.1$, with $Q_{\mathrm{t}}=1 \mathrm{~dB}$, the average rate at the first-hop is slightly higher than that at the second-hop, due to the small energy conversion coefficient at the relay harvesting battery. As $\eta$ increases, i.e., $\eta>0.1$, the average rate at the second hop dominates the average rate at the first hop.

Fig. 9 shows the plots for the average rates versus $Q_{\mathrm{t}}$ with $\eta=0.3$ and $P=1 \mathrm{~dB}$. The figure shows three different rate plots defined by the average rate at the first hop, the average rate at the second hop and the average end-to-end ergodic rate $C$. We notice that $\mathbb{E}\left(R_{1}\right)$ increases rapidly with $Q_{\mathrm{t}}$, while the average rate at the second hop increases slowly with $Q_{\mathrm{t}}$. 




Fig. 9. Average rates (first hop, second hop and end-to-end) versus $Q_{\mathrm{t}}$ at $\eta=0.3$ with $P=1 \mathrm{~dB}$.

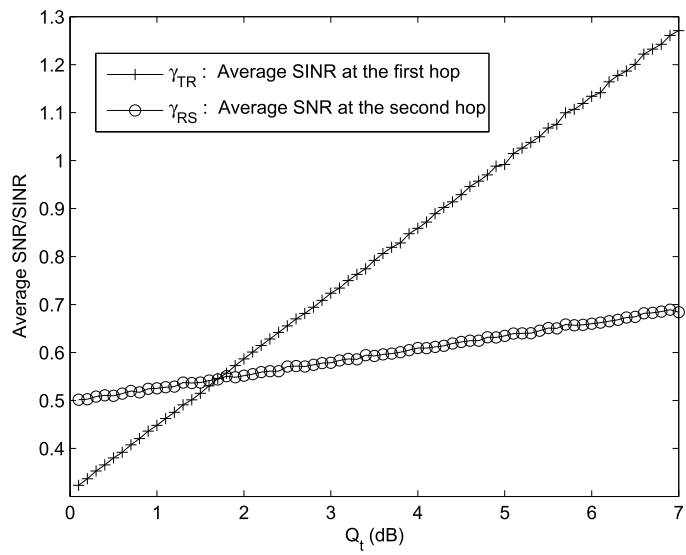

Fig. 10. Average SNR/SINR versus $Q_{\mathrm{t}}$ at $\eta=0.3$ with $P=1 \mathrm{~dB}$.

When $Q_{\mathrm{t}}=0 \mathrm{~dB}$, we observe that the $\mathbb{E}\left(R_{2}\right)$ is higher than $\mathbb{E}\left(R_{1}\right)$. As $Q_{\mathrm{t}}$ increases, e.g., $Q_{\mathrm{t}}>1.5 \mathrm{~dB}$, the average rate at the first hop becomes larger than that at the second hop. This happens due to the fact that in $\mathbb{E}\left(R_{1}\right)$ the transmitter node has a direct access to solar energy, whereas in case of $\mathbb{E}\left(R_{2}\right)$, the harvested power gets affected by the Rayleigh fading channel $h$. Furthermore, the total end-to-end capacity $C$ increases with $Q_{\mathrm{t}}$, showing that the higher the solar energy is, the better the end-to-end ergodic rate is. The numerical results validate the mathematical expression for the ergodic capacity in (38).

Fig. 10 shows the plots for the average SINR at the first-hop and average SNR at the second-hop, versus $Q_{\mathrm{t}}$, at $\eta=0.3$ and $P=1 \mathrm{~dB}$. As observed, both values increase when solar energy increases. Meanwhile, at $Q_{\mathrm{t}}=0 \mathrm{~dB}$, the average SNR at the second hop is higher than the average SINR at the first hop. This is due to the fact that while $Q_{\mathrm{t}}$ is small, i.e., $Q_{\mathrm{t}}=0 \mathrm{~dB}$, the relay still has access to harvesting power from the RF interference signal. At $Q_{\mathrm{t}}=1.5 \mathrm{~dB}$, the two plots coincide each other. Overall, the first-hop SINR increases more rapidly with $Q_{\mathrm{t}}$ compared to the second hop SNR.

Finally, Fig. 11 demonstrates the plots for the average rate at the first hop, the average rate at the second hop, and the average end-to-end ergodic rate $C$, versus the average

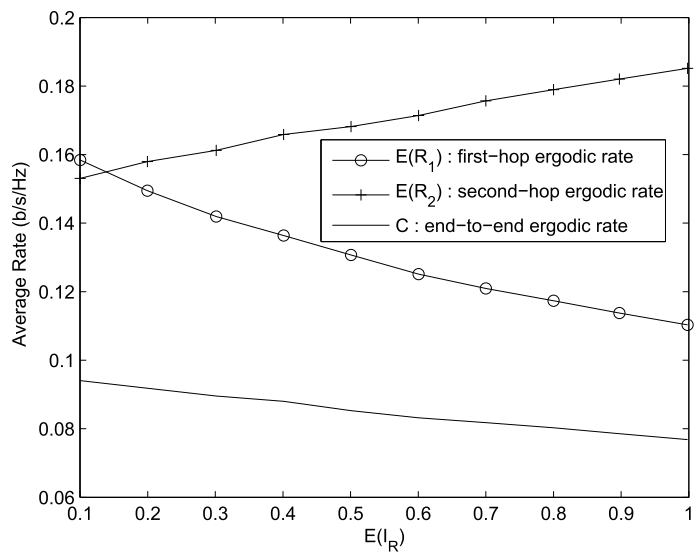

Fig. 11. Average rates (first hop, second hop and end-to-end) versus average interference at the relay $\mathbb{E}\left(I_{R}\right)$, with $\tau=0.4, Q_{\mathrm{t}}=1 \mathrm{~dB}$ and varying $\mu$.

interference power at the relay $\mathbb{E}\left(I_{R}\right)$, with $\eta=0.3, P=1 \mathrm{~dB}$ and $Q_{\mathrm{t}}=1 \mathrm{~dB}$. When $Q_{\mathrm{t}}=1 \mathrm{~dB}$ and the average interference power is small, i.e., $\mathbb{E}\left(I_{R}\right)<0.2$, the first-hop rate is slightly higher than the one at the second hop. The average interference adversely affects the rate of the first hop, therefore $\mathbb{E}\left(R_{1}\right)$ always decreases with $\mathbb{E}\left(I_{R}\right)$; whereas it improves the rate of the second hop through EH. When the interference signal is weak, the rate of the first hop is better than the rate of the second hop. With an increase in the average interference at the relay, $\mathbb{E}\left(R_{2}\right)$ gives higher values since the solar transmitter has access to solar energy. Furthermore, the average end-to-end capacity $C$ shows a monotonic decrease with $\mathbb{E}\left(I_{R}\right)$, revealing that the more the average interference is, the less the end-toend ergodic rate will be.

\section{CONCLUSION}

In this paper, we investigated the system performance of a dual-hop DF relaying system, in which the transmitter and the relay are equipped with fixed, as well as, EH batteries. Harvesting at the transmitter is done through solar energy source, whereas interference from the RF is used as the EH source at the relay. TS is used for EH and information transmission at the relay. We showed that $\mathrm{EH}$ at the source transmitter and at the relay can improve the end-to-end SNR. Closed-form expressions were derived for the CDF of the SNR at each hop, and also for the end-to-end SNR. The analytical expression for the end-to-end ergodic capacity was also obtained. Numerical results were provided to verify the correctness of the closed-from derivations. The effect of energy conversion efficiency and the fixed batteries power levels on the CDF of the SNR for the individual links were also investigated. The results further demonstrated that with the addition of EH at the source and the relay, significant improvement in the system throughput can be achieved. Future work includes consideration of input power constraints at the fixed batteries and catering inter-cell interference and hardware impairments.

\section{APPENDIX A}

Here, we aim to prove the equation in Lemma 1 , given in (1). We start by referring to the below recurrence 
equation [48, p. 101]

$$
\begin{aligned}
\frac{d}{d x} & x^{\alpha} \exp \left(-x-b x^{-1}\right) \\
= & \alpha x^{\alpha-1} \exp \left(-x-b x^{-1}\right)+b x^{\alpha-2} \exp \left(-x-b x^{-1}\right) \\
& -x^{\alpha} \exp \left(-x-b x^{-1}\right)
\end{aligned}
$$

For $\alpha=1$, the expression simplifies to

$$
\frac{d\left(x \exp \left(-x-b x^{-1}\right)\right)}{d x}=\left(1+b x^{-1}-x\right) \times \exp \left(-x-b x^{-1}\right) \text {. }
$$

Re-arranging (40) and taking integration on both sides gives,

$$
\begin{aligned}
\int_{\gamma}^{\infty} & x \exp \left(-x-b x^{-1}\right) d x \\
= & \int_{\gamma}^{\infty} b x^{-1} \exp \left(-x-b x^{-1}\right)+\left.x \exp \left(-x-b x^{-1}\right)\right|_{\gamma} ^{\infty} \\
& +\int_{\gamma}^{\infty} \exp \left(-x-b x^{-1}\right) d x
\end{aligned}
$$

Different parts of (41) can be re-written into generalized incomplete Gamma function [47] as

$$
\begin{aligned}
\int_{\gamma}^{\infty} x \exp \left(-x-b x^{-1}\right) & =\Gamma(2, x ; b), \\
\int_{\gamma}^{\infty} b x^{-1} \exp \left(-x-b x^{-1}\right) & =\Gamma(0, x ; b), \\
\int_{\gamma}^{\infty} \exp \left(-x-b x^{-1}\right) & =\Gamma(1, x ; b) .
\end{aligned}
$$

Hence, replacing (42)-(44) into (41) yields,

$$
\Gamma(2, x ; b)=\Gamma(0, x ; b)+\left.x \exp \left(-x-b x^{-1}\right)\right|_{\gamma} ^{\infty}+\Gamma(1, x ; b) .
$$

\section{REFERENCES}

[1] Z. Hasan, H. Boostanimehr, and V. K. Bhargava, "Green cellular networks: A survey, some research issues and challenges," IEEE Commun. Surveys Tuts., vol. 13, no. 4, pp. 524-540, 4th Quart., 2011.

[2] N. P. Melville, "Information systems innovation for environmental sustainability," MIS Quart., vol. 34, no. 1, pp. 1-21, Mar. 2010.

[3] C. Lange, D. Kosiankowski, R. Weidmann, and A. Gladisch, "Energy consumption of telecommunication networks and related improvement options," IEEE J. Sel. Topics Quantum Electron., vol. 17, no. 2, pp. 285-295, Mar. 2011.

[4] M. Gruber, O. Blume, D. Ferling, D. Zeller, M. A. Imran, and E. C. Strinati, "EARTH-Energy aware radio and network technologies," in Proc. IEEE Int. Symp. Pers. Indoor Mobile Radio Commun. (PIMRC), Tokyo, Japan, Sep. 2009, pp. 1-5.

[5] M. A. Marsan et al., "TREND: Toward real energy-efficient network design," in Proc. Int. Sustain. ICT Sustain. (SustainIT), Pisa, Italy, Oct. 2012, pp. 1-6.

[6] Y. Chen et al., "Fundamental trade-offs on green wireless networks," IEEE Commun. Mag., vol. 49, no. 6, pp. 30-37, Jun. 2011.

[7] K. Lahiri, S. Dey, D. Panigrahi, and A. Raghunathan, "Battery-driven system design: A new frontier in low power design," in Proc. ASP-DAC, Bangalore, India, Jan. 2002, pp. 261-267.

[8] J. Xu and R. Zhang, "Throughput optimal policies for energy harvesting wireless transmitters with non-ideal circuit power," IEEE J. Sel. Areas Commun., vol. 32, no. 2, pp. 322-332, Feb. 2014.

[9] A. J. Goldsmith and S. B. Wicker, "Design challenges for energyconstrained ad hoc wireless networks," IEEE Wireless Commun., vol. 9, no. 4, pp. 8-27, Aug. 2002.
[10] C. K. Ho and R. Zhang, "Optimal energy allocation for wireless communications with energy harvesting constraints," IEEE Trans. Signal Process., vol. 60, no. 9, pp. 4808-4818, Sep. 2012.

[11] A. M. Siddiqui, L. Musavian, and Q. Ni, "Energy efficiency optimization with energy harvesting using harvest-use approach," in Proc. IEEE Int. Conf. Commun. Workshop (ICCW), London, U.K., Jun. 2015, pp. 1982-1987.

[12] S. Hu, Z. Ding, Q. Ni, W. Yu, and Z. Song, "Energy efficiency in energy harvesting cooperative networks with self-energy recycling," in Proc. IEEE Int. Workshop Comput. Aided Modelling Design Commun. Links Netw. (CAMAD), Sep. 2015, pp. 59-63.

[13] A. N. Parks, A. P. Sample, Y. Zhao, and J. R. Smith, "A wireless sensing platform utilizing ambient RF energy," in Proc. IEEE Top. Conf. Biomed. Wireless Technol., Netw., Sensor Syst. (BioWireleSS), Jan. 2013, pp. $154-156$.

[14] P. Harrop and R. Das, "Energy harvesting and storage for electronic devices 2009-2019," IDTechEx, Cambridge, U.K., 1988.

[15] Y. Chen, K. T. Sabnis, and R. A. Abd-Alhameed, "New formula for conversion efficiency of RF EH and its wireless applications," IEEE Trans. Veh. Technol., vol. 65, no. 11, pp. 9410-9414, Nov. 2016.

[16] I. Krikidis, "Simultaneous information and energy transfer in large-scale networks with/without relaying," IEEE Trans. Commun., vol. 62, no. 3, pp. 900-912, Mar. 2014.

[17] Y. Gu and S. Aissa, "Interference aided energy harvesting in decodeand-forward relaying systems," in Proc. IEEE Int. Conf. Commun. (ICC), Sydney, NSW, Australia, Jun. 2014, pp. 5378-5382.

[18] V. R. Cadambe and S. A. Jafar, "Interference alignment and degrees of freedom of the-user interference channel," IEEE Trans. Inf. Theory, vol. 54, no. 8, pp. 3425-3441, Aug. 2008.

[19] L. Liu, R. Zhang, and K.-C. Chua, "Wireless information and power transfer: A dynamic power splitting approach," IEEE Trans. Commun., vol. 61, no. 9, pp. 3990-4001, Sep. 2013.

[20] M. I. Circuits. (2012). Max17710-Energy-Harvesting Charger and Protector Datasheet. Maxim Integrated Circuits, accessed: 2011. [Online]. Available: http://datasheets.maximintegrated.com/en/ds/MAX17710.pdf

[21] H. Ostaffe, "RF-based wireless charging and energy harvesting enables new applications and improves product design," Mouser Electron., Mansfield, TX, USA, 2017. [Online]. Available: https://www.mouser.com/pdfdocs/rf-basedwireless-charging.pdf

[22] N. T. Do, D. B. da Costa, and B. An, "Performance analysis of multirelay RF energy harvesting cooperative networks with hardware impairments," IET Commun., vol. 10, no. 18, pp. 2551-2558, Dec. 2016.

[23] Z. Ding, S. M. Perlaza, I. Esnaola, and H. V. Poor, "Power allocation strategies in energy harvesting wireless cooperative networks," IEEE Trans. Wireless Commun., vol. 13, no. 2, pp. 846-860, Feb. 2014.

[24] P. Grover and A. Sahai, "Shannon meets Tesla: Wireless information and power transfer," in Proc. IEEE Int. Symp. Inf. Theory, Austin, TX, USA, Jun. 2010, pp. 2363-2367.

[25] Z. Wang, V. Aggarwal, and X. Wang, "Optimal energy-bandwidth allocation for energy harvesting interference networks," in Proc. IEEE Int. Symp. Inf. Theory (ISIT), Honolulu, HI, USA, Jun. 2014, pp. 1166-1170.

[26] K. Tutuncuoglu and A. Yener, "Optimum transmission policies for battery limited energy harvesting nodes," IEEE Trans. Wireless Commun., vol. 11, no. 3, pp. 1180-1189, Mar. 2012.

[27] Z. Xiang and M. Tao, "Robust beamforming for wireless information and power transmission," IEEE Wireless Commun. Lett., vol. 1, no. 4, pp. 372-375, Aug. 2012.

[28] H. Tabassum, E. Hossain, A. Ogundipe, and D. I. Kim, "Wirelesspowered cellular networks: Key challenges and solution techniques," IEEE Commun. Mag., vol. 53, no. 6, pp. 63-71, Jun. 2015.

[29] Y. Liu, S. A. Mousavifar, Y. Deng, C. Leung, and M. Elkashlan, "Wireless energy harvesting in a cognitive relay network," IEEE Trans. Wireless Commun., vol. 15, no. 4, pp. 2498-2508, Apr. 2016.

[30] Y. Gu and S. Aissa, "RF-based energy harvesting in decode-and-forward relaying systems: Ergodic and outage capacities," IEEE Trans. Wireless Commun., vol. 14, no. 11, pp. 6425-6434, Nov. 2015.

[31] X. Lu, I. Flint, D. Niyato, N. Privault, and P. Wang, "Performance analysis of simultaneous wireless information and power transfer with ambient RF energy harvesting," in Proc. IEEE Int. Wireless Commun. Netw. Conf. (WCNC), New Orleans, LA, USA, Apr. 2015, pp. 1303-1308.

[32] S. Q. Nguyen et al., "Performance analysis of energy-harvesting relay selection systems with multiple antennas in presence of transmit hardware impairments," in Proc. Int. Conf. Adv. Technol. Commun. (ATC), Hanoi, Vietnam, Oct. 2016, pp. 126-130. 
[33] W. Han, J. Ge, and J. Men, "Performance analysis for NOMA energy harvesting relaying networks with transmit antenna selection and maximal-ratio combining over Nakagami- $m$ fading," IET Commun., vol. 10, no. 18, pp. 2687-2693, Dec. 2016.

[34] Y. Liu, Z. Ding, M. Elkashlan, and H. V. Poor, "Cooperative nonorthogonal multiple access with simultaneous wireless information and power transfer," IEEE J. Sel. Areas Commun., vol. 34, no. 4, pp. 938-953, Apr. 2016.

[35] Y. Chen, "Energy-harvesting AF relaying in the presence of interference and Nakagami-m fading," IEEE Trans. Wireless Commun., vol. 15, no. 2, pp. 1008-1017, Feb. 2016.

[36] E. Illi, F. El Bouanani, and F. Ayoub, "Performance analysis of dual-hop underwater communication system subject to $\mathrm{k}-\mu$ shadowed fading channel," in Proc. IEEE Int. Conf. Adv. Commun. Syst. Inf. Secur. (ACOSIS), Marrakesh, Morocco, Oct. 2016, pp. 1-6.

[37] X. Lu, P. Wang, D. Niyato, D. I. Kim, and Z. Han, "Wireless networks with RF energy harvesting: A contemporary survey," IEEE Commun. Surveys Tuts., vol. 17, no. 2, pp. 757-789, 2nd Quart., 2015.

[38] B. Varan, K. Tutuncuoglu, and A. Yener, "Energy harvesting communications with continuous energy arrivals," in Proc. IEEE, Inf. Theory, Appl. Workshop (ITA), San Diego, CA, USA, Feb. 2014, pp. 1-10.

[39] R. Rajesh, V. Sharma, and P. Viswanath, "Capacity of fading Gaussian channel with an energy harvesting sensor node," in Proc. IEEE Global Commun. Conf. (GLOBECOM), Houston, TX, USA, Dec. 2011, pp. 1-6.

[40] R. Rajesh, V. Sharma, and P. Viswanath, "Capacity of Gaussian channels with energy harvesting and processing cost," IEEE Trans. Inf. Theory, vol. 60, no. 5, pp. 2563-2575, May 2014.

[41] A. Kansal, J. Hsu, S. Zahedi, and M. B. Srivastava, "Power management in energy harvesting sensor networks," ACM Trans. Embedded Comput. Syst., vol. 6, no. 4, p. 32, Sep. 2007.

[42] Y. Liu, L. Wang, S. A. R. Zaidi, M. Elkashlan, and T. Q. Duong, "Secure D2D communication in large-scale cognitive cellular networks: A wireless power transfer model," IEEE Trans. Commun., vol. 64, no. 1, pp. 329-342, Jan. 2016.

[43] M. Xia and S. Aissa, "On the efficiency of far-field wireless power transfer," IEEE Trans. Signal Process., vol. 63, no. 11, pp. 2835-2847, Jun. 2015.

[44] H. Shin and M. Z. Win, "MIMO diversity in the presence of double scattering," IEEE Trans. Inf. Theory, vol. 54, no. 7, pp. 2976-2996, Jul. 2008.

[45] A. Papoulis and S. U. Pillai, Probability, Random Variables, and Stochastic Processes. New York, NY, USA: McGraw-Hill, 2002.

[46] A. Jeffrey and D. Zwillinger, Table of Integrals, Series, and Products. Orlando, FL, USA: Academic, Apr. 2007.

[47] M. A. Chaudhry and S. M. Zubair, "Generalized incomplete Gamma functions with applications," J. Comput. Appl. Math., vol. 55, no. 1, pp. 99-123, Oct. 1994.

[48] M. A. Chaudhry, N. Temme, and E. Veling, "Asymptotics and closed form of a generalized incomplete Gamma function," J. Comput. Appl. Math., vol. 67, no. 2, pp. 371-379, Mar. 1996.

[49] G. P. Beaumont, Probability and Random Variables. New York, NY, USA: Elsevier, 2005

[50] S. Choudhury and J. D. Gibson, "Ergodic capacity, outage capacity, and information transmission over Rayleigh fading channels," in Proc. Inf. Theory Appl. Workshop (ITA), CA, USA, Jan. 2007.

[51] S. Mathew et al., "Dye-sensitized solar cells with $13 \%$ efficiency achieved through the molecular engineering of porphyrin sensitizers," Nature Chem., vol. 6, no. 3, pp. 242-247, Mar. 2014.

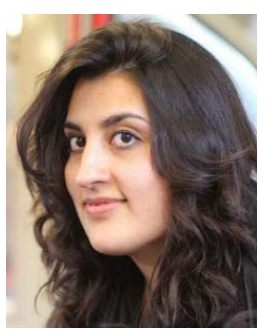

Arooj Mubashara Siddiqui received the B.Sc degree in electrical (telecommunication) engineering from the COMSATS Institute of Information Technology, Pakistan, in 2009, the M.Sc. degree in mobile broadband communications, and the Ph.D. degree in communication systems from the InfoLab21, School of Computing and Communications, Lancaster University, Lancaster, U.K., in 2013 and 2017, respectively. She is currently an Assistant Professor with the Department of Electrical Engineering, COMSATS Institute of Information Technology, Islamabad, Pakistan. Her research interests include Energy Harvesting, energy-efficient transmission techniques and optimization toward 5G Green communications.

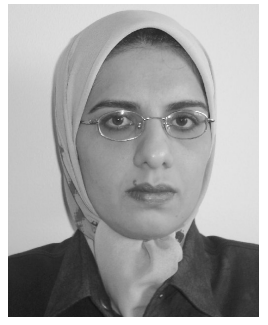

Leila Musavian (S'05-M'07) received the Ph.D. degree in telecommunications from the Kings College London, U.K., in 2006. She was a Lecturer with the InfoLab21, Lancaster University from 2012 to 2016. She was a Research Associate with McGill University from 2011 to 2012 and a PostDoctoral Fellow with INRS-EMT, Canada, from 2006 to 2008. She is currently a Reader with the School of Computer Science and Electronic Engineering, University of Essex. Her research interests lie in radio resource management for next generation wireless networks, CRNs, energy harvesting, green communication, energyefficient transmission techniques, cross-layer design for delay QoS provisioning, and $5 \mathrm{G}$ systems. She is an Editor of the IEEE TRANSACTIONS ON WiRELESS COMMUNiCATIONS, an Executive Editor of the Transactions on Emerging Telecommunications Technologies (Wiley) and an Associate Editor of the Internet Technology Letters (Wiley).

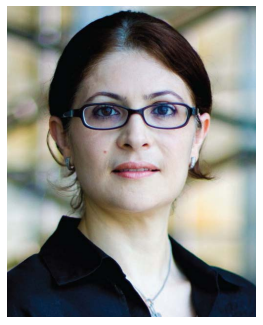

Sonia Aïssa (S'93-M'00-SM'03) received her $\mathrm{Ph} . \mathrm{D}$. degree in Electrical and Computer Engineering from McGill University, Montreal, QC, Canada, in 1998. Since then, she has been with the Institut National de la Recherche Scientifique-Energy, Materials and Telecommunications Center (INRS-EMT), University of Quebec, Montreal, QC, Canada, where she is a Full Professor.

From 1996 to 1997, she was a Researcher with the Department of Electronics and Communications of Kyoto University, and with the Wireless Systems Laboratories of NTT, Japan. From 1998 to 2000, she was a Research Associate at INRS-EMT. In 2000-2002, while she was an Assistant Professor, she was a Principal Investigator in the major program of personal and mobile communications of the Canadian Institute for Telecommunications Research, leading research in radio resource management for wireless networks. From 2004 to 2007, she was an Adjunct Professor with Concordia University, Montreal. She was Visiting Invited Professor at Kyoto University, Japan, in 2006, and Universiti Sains Malaysia, in 2015. Her research interests include the modeling, design and performance analysis of wireless communication systems and networks.

Dr. Aissa is the Founding Chair of the IEEE Women in Engineering Affinity Group in Montreal, 2004-2007; acted as TPC Symposium Chair or Cochair at IEEE ICC '06 '09 '11 '12; Program Cochair at IEEE WCNC 2007; TPC Cochair of IEEE VTC-spring 2013; and TPC Symposia Chair of IEEE Globecom 2014; and serves as TPC Vice-Chair of IEEE Globecom 2018. Her main editorial activities include: Editor, SHAPE IEEE TRANSACTIONS ON WIRELESS COMMUNICATIONS, 2004-2012; Associate Editor and Technical Editor, SHAPE IEEE COMMUNICATIONS MAGAZINE, 2004-2015; Technical Editor, SHAPE IEEE WIRELESS COMMUNICATIONS MAGAZINE, 2006-2010; and Associate Editor, Wiley Security and Communication Networks Journal, 2007-2012. She currently serves as Area Editor for the SHAPE IEEE TRANSACTIONS ON WiRELESS COMMUNiCATIONS. Awards to her credit include the NSERC University Faculty Award in 1999; the Quebec Government FRQNT Strategic Faculty Fellowship in 2001-2006; the INRSEMT Performance Award multiple times since 2004, for outstanding achievements in research, teaching and service; and the Technical Community Service Award from the FRQNT Centre for Advanced Systems and Technologies in Communications, 2007. She is co-recipient of five IEEE Best Paper Awards and of the 2012 IEICE Best Paper Award; and recipient of NSERC Discovery Accelerator Supplement Award. She served as Distinguished Lecturer of the IEEE Communications Society and Member of its Board of Governors in 2013-2016 and 2014-2016, respectively. Professor Aïssa is a Fellow of the Canadian Academy of Engineering.

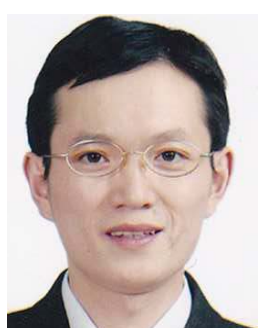

Qiang Ni (M'04-SM'08) received the B.Sc., M.Sc., and Ph.D. degrees from the Huazhong University of Science and Technology, China, all in engineering. $\mathrm{He}$ is a Professor and the Head of Communication Systems Research Group, School of Computing and Communications, InfoLab21, Lancaster University, U.K. He has authored over 180 papers in these areas. He is a Voting Member of the IEEE 1932.1 standard. He was an IEEE 802.11 Wireless Standard Working Group Voting Member and a contributor to various IEEE Wireless Standards. His research interests lie in the area of future generation communications and networking, including green communications, 5G, energy harvesting, cognitive radio, ultra-dense networks, SDN, IoTs, and vehicular networks. 